\title{
Proposal for a trial of early left ventricular venting during venoarterial extracorporeal membrane oxygenation for cardiogenic shock
}

Michael Ibrahim, MD, PhD, Michael A. Acker, MD, Wilson Szeto, MD, Jacob Gutsche, MD, Matthew Williams, MD, Pavan Atluri, MD, Matthew Woods, MD, Thomas Richards, PhD,

Timothy J. Gardner, MD, Jeremy McGarvey, MD, Mark Epler, MD, Joyce Wald, MD, Eduardo Rame, MD, Edo Birati, MD, and Christian Bermudez, MD

\section{ABSTRACT}

Objective: Patients with profound cardiogenic shock may require venoarterial (VA) extracorporeal membrane oxygenation (ECMO) for circulatory support most commonly via the femoral vessels. The rate of cardiac recovery in this population remains low, possibly because peripheral VA-ECMO increases ventricular afterload. Whether direct ventricular unloading in peripheral VA-ECMO enhances cardiac recovery is unknown, but is being more frequently utilized. A randomized trial is warranted to evaluate the clinical effectiveness of percutaneous left ventricle venting to enhance cardiac recovery in the setting of VA-ECMO.

Methods: We describe the rationale, design, and initial testing of a randomized controlled trial of VA-ECMO with and without percutaneous left ventricle venting using a percutaneous micro-axial ventricular assist device.

Results: This is an ongoing prospective randomized controlled trial in adult patients with primary cardiac failure presenting in cardiogenic shock requiring peripheral VA-ECMO, designed to test the safety and effectiveness of percutaneous left ventricle venting in improving the rate of cardiac recovery.

Conclusions: The results of this nonindustry-sponsored trial will provide critical information on whether left ventricle unloading in peripheral VA-ECMO is safe and effective. (JTCVS Open 2021;8:393-400)

From the Division of Cardiovascular Surgery, Department of Surgery, Hospital of the University of Pennsylvania, The University of Pennsylvania, Philadelphia, Pa. Received for publication Sept 29, 2020; accepted for publication July 26, 2021; available ahead of print Aug 14, 2021.

Address for reprints: Michael Ibrahim, MD, PhD, Division of Cardiovascular Surgery, Department of Surgery, Hospital of the University of Pennsylvania, The University of Pennsylvania, 3400 Spruce St, 6 Silverstein Pavilion, Philadelphia, PA 19104 (E-mail: michael.ibrahim@uphs.upenn.edu).

2666-2736

Copyright (C) 2021 The Author(s). Published by Elsevier Inc. on behalf of The American Association for Thoracic Surgery. This is an open access article under the CC BY-NC-ND license (http://creativecommons.org/licenses/by-nc-nd/4.0/).

https://doi.org/10.1016/j.xjon.2021.07.024

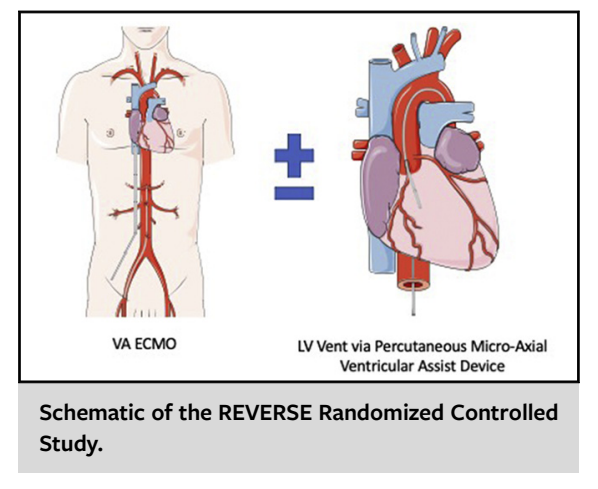

CENTRAL MESSAGE

REVERSE tested the hypothesis

that early unloading using a

percutaneous micro-axial ven-

tricular assist device will promote

ventricular recovery and improve

survival in patients requiring

VA-ECMO.

\section{PERSPECTIVE}

Whereas veno-arterial ECMO provides robust circulatory support, it does not provide a reliable platform for ventricular support due to the lack of volume unloading and increased LV afterload. The REVERSE study is the first randomized study of the influence of direct ventricular unloading on left ventricular recovery in man.

See Commentaries on pages 401 and 403 .

Venoarterial extra-corporeal membrane oxygenation (VAECMO) is indicated as a hemodynamic rescue strategy in decompensated acute or chronic heart failure presenting as cardiogenic shock. ${ }^{1}$ It has been used across etiologies, including postmyocardial infarction, dilated cardiomyopathy, acute miocarditis, and in postcardiotomy shock. VA-ECMO has a number of effects on the circulation, including improved end-organ perfusion and possibly improved coronary perfusion, and is a bridge to further therapies such as permanent advanced mechanical circulatory support, cardiac transplantation, and to cardiac recovery. The use of VA- 


\section{Abbreviations and Acronyms \\ LVAD $=$ left ventricular assist device \\ VA-ECMO $=$ venoarterial extracorporeal membrane oxygenation}

ECMO has increased substantially over the past several years, ${ }^{2,3}$ but the rate of cardiac recovery observed remains low. ${ }^{4,5}$

Left ventricular assist devices (LVADs) provide longterm mechanical circulatory support and also profoundly mechanically unload the left ventricle. ${ }^{6}$ Multiple clinical studies have documented cardiac recovery using LVAD therapy, with a rate between $10 \%$ and $60 \%$ in selected populations. ${ }^{6-12}$ A large body of basic and clinical science has documented the pivotal role of mechanical load in determining ventricular contractile performance across species (amongst others ${ }^{13-23}$ ). Both clinical data and basic laboratory data support the notion that profound ventricular unloading may result in improved cardiac performance through a variety of mechanisms ranging from triggered de novo cardiomyocyte proliferation, ${ }^{14}$ subcellular calcium handling reverse remodeling, ${ }^{22}$ changes to the extracellular matrix of the myocardium, ${ }^{24}$ reverse remodeling of the neurohormonal milieu, ${ }^{25}$ amongst many others. ${ }^{18,26,27}$ In chronic heart failure, direct ventricular unloading is critical to cardiac recovery. ${ }^{9,10,12,26}$

Among the major deficiencies of peripheral VA-ECMO is the afterload it presents to the $\mathrm{LV}$, with associated ventricular distension $^{28,29}$ and pulmonary congestion, which can derail clinical improvement and hamper cardiac recovery. These conditions can result in a congested, pressure-overloaded ventricle, even in the absence of echocardiographic ventricular distension. This may be ameliorated with the addition of ventricular mechanical unloading, an $L V$ vent, of which there are several varieties, including surgical vents in the LV apex, pulmonary artery vents, and percutaneous transvalvular vents. Among the most popular and least invasive modalities is the Impella pump (Abiomed, Mass), which is a percutaneous micro-axial LVAD available in a number of sizes, providing different flow capacities. Other attractive options include the use of percutaenous pulmonary arterial drainage including ProTek Duo (LivaNova, London, England). Retrospective analyses suggest that the addition of ventricular drainage strategies to reduce ventricular loading in VA-ECMO results in improved survival and recovery of ventricular performance in the setting of cardiogenic shock. ${ }^{5}$ In a number of small studies, the use of additional means to unload the ventricle, results in cardiac recovery and less ventricular distension. ${ }^{30-32}$

A randomized trial to evaluate the safety and effectiveness of percutaneous LV venting in peripheral VA-ECMO is warranted and indeed necessary to guide the care of this population of patients.

\section{Trial Objectives}

The objective of this randomized study is to determine whether or not the addition of early direct ventricular unloading using a percutaneous micro-axial LVAD leads to higher rates of cardiac recovery, defined as survival free from mechanical circulatory support, heart transplantation, or inotropic support at 45 days. This study will also examine the clinical, biochemical, echocardiographic, and radiologic effects of VA-ECMO with and without the addition of percutaneous microaxial LVADs to directly vent the LV to address adjunct important questions such as the effects on pulmonary congestion.

\section{Trial Design}

This is a prospective, multisite, single health system randomized, controlled trial conducted primarily at the University of Pennsylvania. Patients placed on VA-ECMO for cardiogenic shock are screened and once enrolled, randomly assigned 1:1 to either VA-ECMO alone or VAECMO with the addition of a percutaneous micro-axial LVAD. The enrollment period is estimated to be 48 months and all patients will be followed through to hospital discharge and beyond for at least 1 year. The primary end point is 45-day survival free from mechanical circulatory support, heart transplant, or inotropes.

Due to the nature of the interventions, it is not possible to blind patients or investigators. All data will be blinded before analyses. Similarly, informed consent was obtained from the appropriate family members or next of kin, due to the inability to provide consent by participants directly. Serious adverse events are adjudicated by a central committee. An institutional review board review was submitted and obtained (The University of Pennsylvania No. 82198; August 20, 2018).

\section{Crossover}

In the presence of radiologic evidence of severe pulmonary congestion, crossover from the nonvented to vented (percutaneous micro-axial LVAD) arm will be allowed at the discretion of the principal investigator. This consideration is made because the standard of clinical care is that in the presence of severe pulmonary edema, most physicians would advocate addition of an LV vent (surgical or percutaneous) for VA-ECMO patients. In our early experience of 17 patients, only 1 patient crossed over, indicating this will likely be a low-frequency event. To mitigate its influence upon the study, several steps have been taken, including defining need to cross over as a predetermined secondary outcome and including both intention-to-treat and as-treated arms analysis of the primary outcome. Given the significant clinical indications for crossover, the ability to cross 
over, although we anticipate this being a rare event, is important for the safety and clinical acceptability of this study. Information on the data safety monitoring board and central adjudication committee are presented in Appendix 1.

\section{Patient Population}

The population for this trial consists of patients with significant cardiogenic shock despite vasoactive pharmacologic support and requiring VA-ECMO according to institutional criteria (See Online Data Supplement). Following initiation of VA-ECMO according to institutional candidacy guidelines, patients were screened for inclusion in the study. These inclusion criteria are described in Figure 1. They target a population with severe cardiac failure, either acute or acute on chronic decompensation. They span a multitude of common etiologies of heart failure. The ideal patient has acute cardiogenic shock; for example, postmyocardial infarction but without major comorbidities. The exclusion criteria in Figure 1 aim to remove patients with little chance of recovery of cardiac function (eg, those with extremely dilated LV and a pre-ECMO, pre-event ejection fraction $<25 \%$ ), primarily noncardiac shock, and those with contraindications to the percutaneous microaxial LVAD.

\section{Randomization}

Patients must be enrolled and randomized within 12 hours of ECMO initiation. Once randomized, an additional 12 hours to place the device is allowed in the case that the patient is allocated to the treatment arm.

The study design is both covariate balanced and response adaptive. ${ }^{33}$ Patients are randomized, in groups of 10 , to either VA-ECMO alone (VA arm) or VA $+\mathrm{V}$ percutaneous micro-axial LVAD (VA + V arm), as follows:

The first groups of 10 patients (20 patients total) will be assigned to VA arm or VA $+\mathrm{V}$ arm with equal allocation probabilities (flips of a fair coin);

After 45-day responses are observed for the first 2 groups (20 patients total), posterior probabilities of superiority of $\mathrm{VA}$ or VA $+\mathrm{V}$ will be computed, conditional upon the data observed so far. This will be as detailed below.

Using groups of size 10 with an early-stopping rule, we implement for treatment groups labelled VA and VA $+\mathrm{V}$, for convenience, design points D1 through D7:

- D1. Until 45-day responses are observed for the first 2 groups of 10 patients each (20 patients overall), employ equal allocation probabilities to treatments VA and $\mathrm{VA}+\mathrm{V}$.

- D2. After the first 20, 45-day responses are observed, compute the posterior probabilities that VA or VA $+\mathrm{V}$

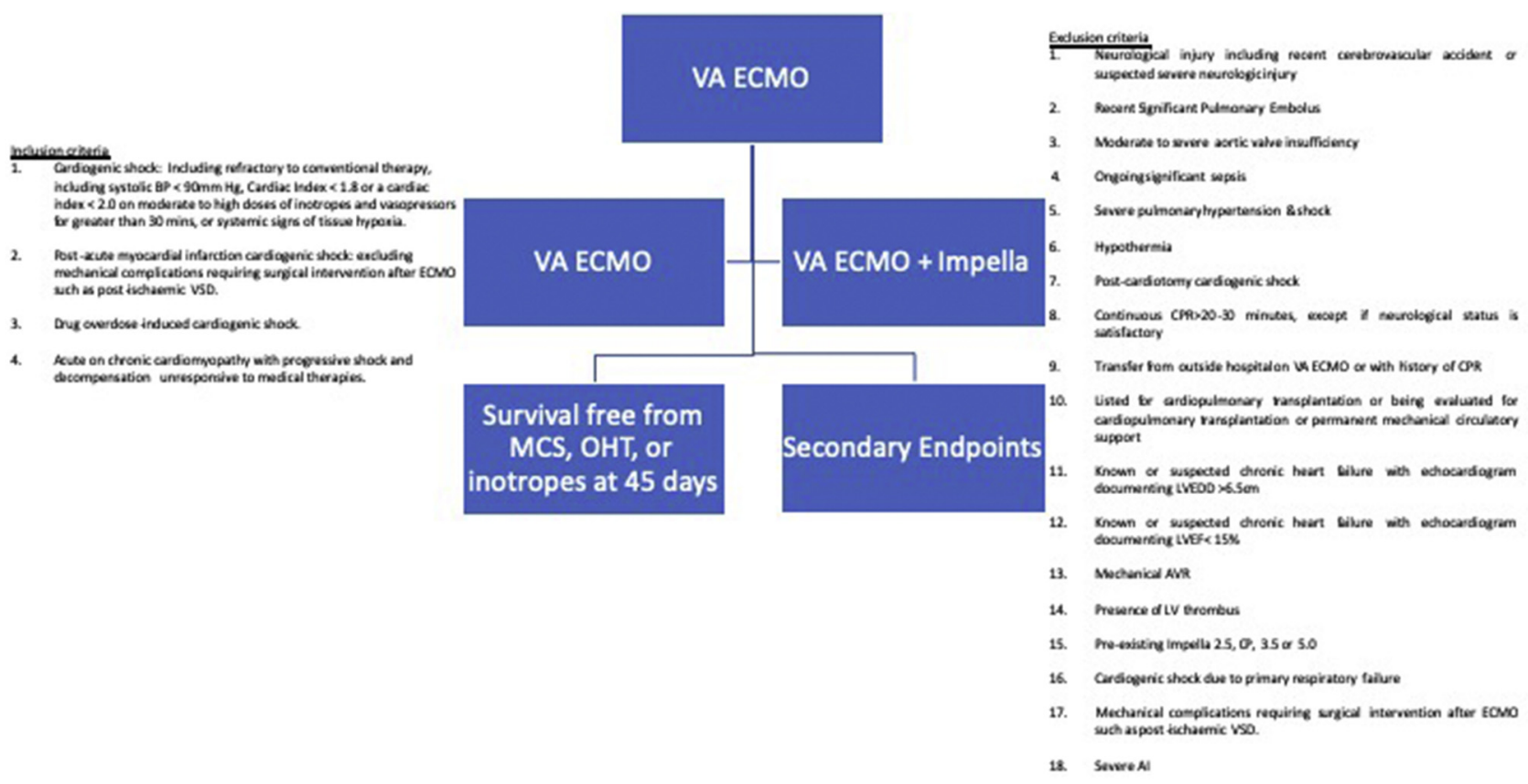

FIGURE 1. Inclusion criteria, visual flowsheet of study, and exclusion criteria for the trial of early left ventricular venting during venoarterial extracorporeal membrane oxygenation for cardiogenic shock study. ECMO, Extracorporeal membrane oxygenation; VSD, ventricular septal defect; VA-ECMO, venoarterial extracorporeal membrane oxygenation; $M C S$, mechanical circulatory support; $O H T$, orthotopic heart transplpant; $C P R$, cardiopulmonary resuscitation; $L V E D D$, left ventricular end diastolic dimension; $L V E F$, left ventricular ejection fraction; $A V R$, aortic valve replacement; $L V$, left ventricle; $A I$, aortic insufficiency. 
is superior, conditional upon the data observed so far in the trial. If either probability exceeds $99 \%$, then stop. Otherwise, proceed to D3.

- D3. After the first 20 responses are observed, fit a logistic model using those 20 responses and their prognostic factors. This model yields a prognostic score for each patient in the third group of 10 patients to be randomized.

- D4. For each patient randomized in the third group of 10 patients, compute the covariate balance probabilities pc_VA and pc_VA $+\mathrm{V}$, as follows: pc_VA $=0.75$ or 0.25 , respectively, according to whether assignment of the patient to VA yields a smaller, or larger, Kolmogorov-Smirnov statistic for testing imbalance of prognostic scores between treatments, and pc_VA $+\mathrm{V}=1-$ pc_VA. The quantities pc_VA and pc_VA $+\mathrm{V}$ bias allocation toward balance on prognostic factors. In case of a tie, set pc_VA $=p_{-} c_{-} V A+V=0.5$.

- D5. For each patient randomized in the third group of 10 patients, compute the response adaptive probabilities pr_VA and pr_VA $+\mathrm{V}$, as follows: pr_VA equals the normalized square-root-transformed probability that VA is the better treatment, based on all responses observed so far in the trial, and pr_VA $+\mathrm{V}=1-\mathrm{pr}_{-} \mathrm{VA}$.

- D6. Randomize each patient in the third group of 10 patients to treatment VA with probability p_VA, equal to $\mathrm{pc} \_\mathrm{VA} \times$ pr_VA $/\left(\mathrm{pc} \_\mathrm{VA} \times\right.$ pr_VA + pc_VA $+\mathrm{V} \times$ pr_VA $+\mathrm{V})$, and to treatment $\mathrm{VA}+\mathrm{V}$ with probability p_VA $+\mathrm{V}=1-\mathrm{p} \_$VA. Note that this revised assignment probability addresses both covariate balance and response adaptation.

- D7. Repeat steps D2 through D6 for each group of 10 patients, but fitting all models using all accumulated responses and prognostic factors, not just the previous block, until either the early stopping criterion is met or the conventional total sample size of 96 patients is reached.

This design ensures that the trial arms will be relatively balanced on the prognostic factors, with simulations showing that fewer than $5 \%$ of potential prognostic factors exhibit significant imbalance. Further, the stopping rule ensures that the trial will likely be stopped in the case that one treatment vastly outstrips the other. Simulations indicate that for effect sizes on the order of those observed by Pappalardo and colleagues, ${ }^{5}$ the expected total sample size can range from 71 to 87 with statistical power exceeding $80 \%$.

\section{End Points}

The primary end point is survival free from inotropes, mechanical circulatory support, or transplantation at 45 days. We selected the 45-day time point because retrospective review of our VA-ECMO experience showed that 30 days was often too early to capture a definitive clinical end point, while 45 days appeared to more rigorously capture true early clinical recovery.
The durability of weaning is captured in a series of other secondary outcomes, including survival to hospital discharge; cardiac recovery, defined as freedom from inotropic support, mechanical circulatory support and transplantation for $>24$ hours; significant between-group differences in inotropic score, pulmonary compliance, and radiologic measures of pulmonary congestion at 24 to 72 hours; significant between-group differences in echocardiographic measurements, biochemical profile, and hemodynamics parameters; and incidence of crossover (Figure 2).

\section{Treatment Intervention}

The standard ECMO setup routinely used at the University of Pennsylvania will be employed. This typically involves a $22 \mathrm{Fr}$ to $25 \mathrm{Fr}$ venous cannula in the femoral vein and a $15 \mathrm{Fr}$ to $19 \mathrm{Fr}$ arterial cannula in the femoral artery. A distal arterial perfusion cannula is uniformly used, often a micropuncture $5 \mathrm{Fr}$ to $9 \mathrm{Fr}$ perfusion cannula. Central aortic and venous cannulation will not be used. A centrifugal pump such as Maquet Rotaflow or Centrimag platform are used on a console with standard Maquet bioline heparin bonded cannulae and Quadrox D oxygenator (Getinge, Sweden).

The percutaneous micro-axial LVAD system that provides a low profile through a $16 \mathrm{Fr}$ sheath with flow up to 3.0 to $3.5 \mathrm{~L} / \mathrm{min}$ on a $15 \mathrm{Fr}$ motor pump setup. The maximum outer diameter of the device as it crosses the iliofemoral subclavian vessels is $5.8 \mathrm{~mm}$. In the absence of significant peripheral vascular disease, it will be inserted via femoral artery using the Seldinger or open surgical technique in the operating theatre or cardiac catheterization lab under fluoroscopic guidance, with its position readjusted under echocardiographic guidance. The subclavian artery route is also well established and will be used when more appropriate than femoral access. Angiography will be used routinely to guide placement. The device catheter will be positioned near the LV apex with the distal end of the catheter between 3 and $4 \mathrm{~cm}$ from the aortic valve.

In addition, wherever possible, all patients will receive a Swan-Ganz catheter, which is the standard of care in the management of cardiogenic shock.

Our full protocol details heparinization protocols and target activated partial thromboplastin clotting time goals.

\section{Follow-up}

It is essential to assess the long-term durability of recovery in survivors of both the control and experimental arms of the study. We propose to follow up at $3,6,9$, and 12 months. This includes a visit with a heart failure cardiologist for a clinical history and physical examination, SF-36 quality of life questionnaire, transthoracic echocardiogram. We would also draw a single sample of peripheral venous blood for storage in a heart failure biomarker bank. At 


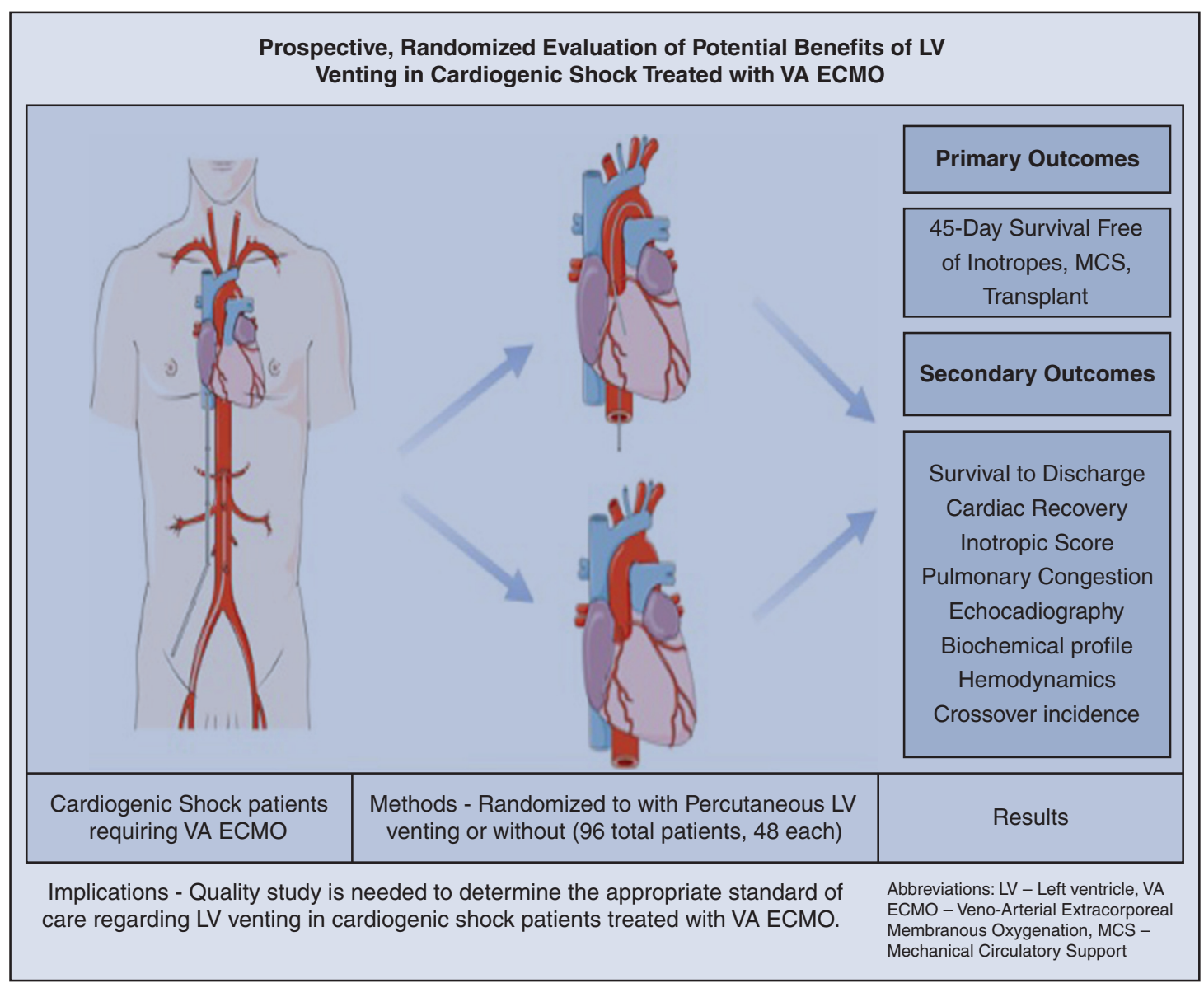

FIGURE 2. Visual representation of the Proposal for a trial of early left ventricular venting during venoarterial extracorporeal membrane oxygenation for cardiogenic shockstudy showing patient population, randomization to either left ventricle venting group or without, and list of primary and secondary outcomes.

6 months, per the standard of care, patients would undergo submaximal cardiopulmonary exercise testing using the 6minute walk test with measurement of maximal oxygen consumption.

\section{Sample Size, Power, and Analysis}

Using binary response rates at 45 days based on recent work by Pappalardo and colleagues, ${ }^{5}$ statistical power exceeds $80 \%$ at $\alpha=0.05$ to detect a doubling of the response rate, with sample sizes of 48 VA-ECMO and 48 VA-ECMO plus percutaneous micro-axial LVAD patients.

The data analysis plan for testing the primary hypothesis of whether VA $+\mathrm{V}$ is superior to VA involves the Bayesian fitting of logistic regression and Cox proportional hazards models for outcome at 45-days follow-up, with a binary indicator variable of treatment arm and adjustment for potential confounders not included in the design. ${ }^{29}$ Secondary end point of survival to discharge and cardiac recovery will be addressed similarly. Group differences in inotropic scores and other continuous measures will be tested by analysis of variace, whereas group effects on categorical outcomes will be tested by contingency tables.

\section{Additional Analyses}

Additional analyses include using logistic regression and Cox models to relate patient-level hemodynamic parameters to survival outcomes.

\section{DISCUSSION}

The trend in ECMO mortality has remained relatively stable and high, with approximately $50 \%$ to $60 \%$ inhospital mortality, ${ }^{3}$ and even $80 \%$ in some series. ${ }^{5}$ Undoubtedly, this is related to the underlying level of critical illness in this population. Likewise, the rate of weaning from VA-ECMO is low. ${ }^{4,5}$ Recently, the influence of peripheral VA-ECMO on human ventricles has been described in multiple case series, showing increased LV diameters consistent with the physiological influence of retrograde perfusion, which may be ameliorated by direct unloading. ${ }^{28,30,32}$ This may be a critical barrier to cardiac recovery using this platform.

The notion of unloading or resting the ventricle as a stimulus to cardiac recovery is not new: in 1945, Samuel Levine stated that "the first principle in the treatment of such a

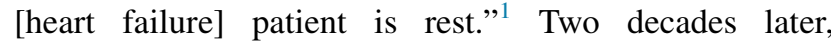


McDonald and colleagues, using this principle to treat patients with congestive heart failure, reported that "with maximal reduction in the workload of the dilated heart by prolonged bed rest one can often achieve some degree of recovery (reviewed in 26)."2 The development of direct mechanical unloading has allowed us to fully explore this potential and over the past several decades, a large body of basic and clinical science has shown that direct ventricular unloading is the cornerstone of recovering the LV by mechanical means. ${ }^{26}$ Clinical recovery from even severe chronic dilated cardiomyopathy has been documented in selected patients at a rate of up to $60 \%,{ }^{10}$ and at lower rates in multiple series. ${ }^{26}$ This has triggered an intensive scientific exploration of the influence of mechanical unloading on a failing mammalian ventricle. Mechanical unload triggers load-specific molecular pathways triggering changes as diverse as reactivation of the fetal genome, reorganization of the cell membrane, de novo cellular formation, and changes to tissue architecture and mass.

VA-ECMO has emerged as the primary mechanical rescue therapy for acute cardiogenic shock, and its use has increased substantially over the past several years. $^{2,3,34,35}$ Therefore, we must understand what platform maximizes the chances of cardiac recovery. The addition of direct ventricular unloading with a percutaneous LVAD is an attractive option due to its availability, ease of implantation, and relatively low morbidity profile. As stated, some retrospective data suggest addition of these devices can augment survival and recovery ${ }^{5}$; and so this is an opportune moment to test this in the form of the prospective randomized study we here describe. Additionally, this study will provide great insight into the effect of these treatment modalities on clinically important measures such as pulmonary artery pressure, pulmonary edema, renal function, inotrope requirements, liver function, acidosis, and many others.

Recruitment will be a challenge in this trial, even across the clinically busy network of Penn hospitals and possibly partners. Families being approached are in a state of crisis, these events take place at all hours and these patients are new to us, often being transferred or coming in through the emergency department and so it is a challenge to understand their eligibility. In other surgical trials, the volume of the target population was, in reality, considerably lower than anticipated $^{36}$ and other barriers to recruitment emerged. This is even more problematic in the setting of a surgical shock trial. Nevertheless, we remain committed to the completion of this study.

The clinical implications for practice of this study will be several. First, we are studying a true area of clinical equipoise-how does the risk and cost of additional intervention compare with the efficacy of direct ventricular unloading. Hitherto, this question has been informed by each physicians' experiential bias but without the benefit of good, prospective, and randomized data. This trial has something to say about the paradigm for ventricular recovery in cardiogenic shock and indeed would be the first true randomized test of the concept of mechanical unloading as a stimulus for ventricular recovery. We have defined a challenging and strict criteria for the primary outcome, which includes survival. It will also be important to understand whether or not there is a ventricle-level effect because survival may be influenced by the overall acuity and morbidity of the population.

\section{CONCLUSIONS}

We believe that this trial will be an informative study that will provide rich data and clinical evidence for an emerging treatment modality that has significant promise but remains to be tested rigorously.

\section{Conflict of Interest Statement}

The authors reported no conflicts of interest.

The Journal policy requires editors and reviewers to disclose conflicts of interest and to decline handling or reviewing manuscripts for which they may have a conflict of interest. The editors and reviewers of this article have no conflicts of interest.

\section{References}

1. Peura JL, Colvin-Adams M, Francis GS, Grady KL, Hoffman TM, Jessup M, et al. Recommendations for the use of mechanical circulatory support: device strategies and patient selection: a scientific statement from the American Heart Association. Circulation. 2012;126:2648-67.

2. Stentz MJ, Kelley ME, Jabaley CS, O'Reilly-Shah V, Groff RF, Moll V, et al. Trends in extracorporeal membrane oxygenation growth in the United States, 2011-2014. ASAIO J. 2019;65:712-7.

3. McCarthy FH, McDermott KM, Kini V, Gutsche JT, Wald JW, Xie D, et al. Trends in US extracorporeal membrane oxygenation use and outcomes: 20022012. Semin Thorac Cardiovasc Surg. 2015;27:81-8.

4. Cheng YT, Garan AR, Sanchez J, Kurlansky P, Ando M, Cevasco M, et al. Midterm outcomes of bridge-to-recovery patients after short-term mechanical circulatory support. Ann Thorac Surg. 2019;108:524-30.

5. Pappalardo F, Schulte C, Pieri M, Schrage B, Contri R, Soeffker G, et al. Concomitant implantation of Impella on top of veno-arterial extracorporeal membrane oxygenation may improve survival of patients with cardiogenic shock. Eur J Heart Fail. 2017;19:404-12.

6. Terracciano CM, Miller LW, Yacoub MH. Contemporary use of ventricular assist devices. Annu Rev Med. 2010;61:255-70.

7. Maybaum S, Mancini D, Xydas S, Starling RC, Aaronson K, Pagani FD, et al. Cardiac improvement during mechanical circulatory support: a prospective multicenter study of the LVAD working group. Circulation. 2007;115:2497-505.

8. Terracciano CM, Hardy J, Birks EJ, Khaghani A, Banner NR, Yacoub MH. Clinical recovery from end-stage heart failure using left-ventricular assist device and pharmacological therapy correlates with increased sarcoplasmic reticulum calcium content but not with regression of cellular hypertrophy. Circulation. 2004;109:2263-5.

9. Birks EJ, Hall JL, Barton PJ, Grindle S, Latif N, Hardy JP, et al. Gene profiling changes in cytoskeletal proteins during clinical recovery after left ventricularassist device support. Circulation. 2005;112:157-64.

10. Birks EJ, Tansley PD, Hardy J, George RS, Bowles CT, Burke M, et al. Left ventricular assist device and drug therapy for the reversal of heart failure. $N$ Engl J Med. 2006;355:1873-84.

11. Hall JL, Birks EJ, Grindle S, Cullen ME, Barton PJ, Rider JE, et al. Molecular signature of recovery following combination left ventricular assist device (LVAD) support and pharmacologic therapy. Eur Heart J. 2007;28:613-27. 
12. Birks EJ, George RS, Hedger M, Bahrami T, Wilton P, Bowles CT, et al. Reversal of severe heart failure with a continuous-flow left ventricular assist device and pharmacological therapy: a prospective study. Circulation. 2011;123:381-90.

13. Oriyanhan W, Tsuneyoshi H, Nishina T, Matsuoka S, Ikeda T, Komeda M. Determination of optimal duration of mechanical unloading for failing hearts to achieve bridge to recovery in a rat heterotopic heart transplantation model. $J$ Heart Lung Transplant. 2007;26:16-23.

14. Canseco DC, Kimura W, Garg S, Mukherjee S, Bhattacharya S, Abdisalaam S, et al. Human ventricular unloading induces cardiomyocyte proliferation. J Am Coll Cardiol. 2015;65:892-900.

15. Klotz S, Burkhoff D, Garrelds IM, Boomsma F, Danser AH. The impact of left ventricular assist device-induced left ventricular unloading on the myocardial renin-angiotensin-aldosterone system: therapeutic consequences? Eur Heart J. 2009;30:805-12.

16. Frazier OH, Benedict CR, Radovancevic B, Bick RJ, Capek P, Springer WE, et al. Improved left ventricular function after chronic left ventricular unloading. Ann Thorac Surg. 1996;62:675-81.

17. Bick RJ, Grigore AM, Poindexter BJ, Schnee PM, Nussmeier NA, Gregoric ID, et al. Left ventricular unloading with an assist device results in receptor relocalization as well as increased beta-adrenergic receptor numbers: are these changes indications for outcome? J Card Surg. 2005;20:332-6.

18. Ibrahim M, Rao C, Athanasiou T, Yacoub MH, Terracciano CM. Mechanical unloading and cell therapy have a synergistic role in the recovery and regeneration of the failing heart. Eur J Cardiothorac Surg. 2012;42:312-8.

19. Klotz S, Foronjy RF, Dickstein ML, Gu A, Garrelds IM, Danser AH, et al. Mechanical unloading during left ventricular assist device support increases left ventricular collagen cross-linking and myocardial stiffness. Circulation. 2005;112: 364-74.

20. Schwoerer AP, Neuber C, Schmechel A, Melnychenko I, Mearini G, Boknik P, et al. Mechanical unloading of the rat heart involves marked changes in the protein kinase-phosphatase balance. J Mol Cell Cardiol. 2008;45:846-52.

21. Ogletree-Hughes ML, Stull LB, Sweet WE, Smedira NG, McCarthy PM, Moravec CS. Mechanical unloading restores beta-adrenergic responsiveness and reverses receptor downregulation in the failing human heart. Circulation. 2001;104:881-6.

22. Ibrahim M, Navaratnarajah M, Siedlecka U, Rao C, Dias P, Moshkov AV, et al. Mechanical unloading reverses transverse tubule remodelling and normalizes local $\mathrm{Ca}(2+)$-induced $\mathrm{Ca}(2+)$ release in a rodent model of heart failure. Eur $J$ Heart Fail. 2012;14:571-80.

23. Drakos SG, Terrovitis JV, Nanas JN, Charitos EI, Ntalianis AS, Malliaras KG, et al. Reverse electrophysiologic remodeling after cardiac mechanical unloading for end-stage nonischemic cardiomyopathy. Ann Thorac Surg. 2011;91:764-9.

24. Bruggink $\mathrm{AH}$, van Oosterhout $\mathrm{MF}$, de Jonge $\mathrm{N}$, Ivangh B, van Kuik J, Voorbij RH, et al. Reverse remodeling of the myocardial extracellular matrix after prolonged left ventricular assist device support follows a biphasic pattern. J Heart Lung Transplant. 2006;25:1091-8.

25. Thompson LO, Skrabal CA, Loebe M, Lafuente JA, Roberts RR, Akgul A et al. Plasma neurohormone levels correlate with left ventricular functiona and morphological improvement in LVAD patients. J Surg Res. 2005;123: 25-32.

26. Ibrahim M, Yacoub MH. Bridge to recovery and weaning protocols. Heart Fail Clin. 2014;10:S47-55.

27. Ibrahim M, Terracciano C, Yacoub MH. Can bridge to recovery help to reveal the secrets of the failing heart? Curr Cardiol Rep. 2012;14:392-6.

28. Lim HS. The effect of Impella CP on cardiopulmonary physiology during venoarterial extracorporeal membrane oxygenation support. Artif Organs. 2017; 41:1109-12.

29. Seo T, Ito T, Iio K, Kato J, Takagi H. Experimental study on the hemodynamic effects of veno-arterial extracorporeal membrane oxygenation with an automatically driven blood pump on puppies. Artif Organs. 1991;15: 402-7.

30. Cheng A, Swartz MF, Massey HT. Impella to unload the left ventricle during peripheral extracorporeal membrane oxygenation. ASAIO J. 2013;59:533-6.

31. Kawashima D, Gojo S, Nishimura T, Itoda Y, Kitahori K, Motomura N, et al. Left ventricular mechanical support with Impella provides more ventricular unloading in heart failure than extracorporeal membrane oxygenation. ASAIO J. 2011;57: 169-76.

32. Koeckert MS, Jorde UP, Naka Y, Moses JW, Takayama H. Impella LP 2.5 for left ventricular unloading during venoarterial extracorporeal membrane oxygenation support. J Card Surg. 2011;26:666-8.

33. Yuan Y, Huang X, Liu S. A Bayesian response-adaptive covariate-balanced randomization design with application to a leukemia clinical trial. Stat Med. 2011;30:1218-29.

34. Esper SA, Bermudez C, Dueweke EJ, Kormos R, Subramaniam K, Mulukutla S, et al. Extracorporeal membrane oxygenation support in acute coronary syndromes complicated by cardiogenic shock. Catheter Cardiovasc Interv. 2015; 86(Suppl 1):S45-50.

35. Bermudez CA, Rocha RV, Toyoda Y, Zaldonis D, Sappington PL, Mulukutla S, et al. Extracorporeal membrane oxygenation for advanced refractory shock in acute and chronic cardiomyopathy. Ann Thorac Surg. 2011;92:2125-31.

36. Smith PK, Michler RE, Woo YJ, Alexander JH, Puskas JD, Parides MK, et al Design, rationale, and initiation of the surgical interventions for moderate ischemic mitral regurgitation trial: a report from the cardiothoracic surgical trials network. J Thorac Cardiovasc Surg. 2012;143:111-7. 117.e1.

Key Words: cardiac recovery, cardiogenic shock 


\section{APPENDIX 1. DATA SAFETY MONITORING BOARD AND CENTRAL ADJUDICATION COMMITTEE}

This study has also incorporated the use of a data safety monitoring board (DSMB) an a central adjudication committee (CAC) to oversee study progression, to protect the integrity of the investigation, and most importantly, to protect the study's vulnerable study population.

The DSMB will be composed of an independent panel of clinicians and scientists considered experts in the fields of cardiogenic shock, mechanical circulatory support, biostatistics, and clinical trial design and performance. Although care will be taken to appoint a comprehensive collection of expertise, allowances will be made to accommodate input from nonvoting ad hoc specialists on an asneeded basis. Potential members will be vetted for their suitability and expertise, as well as to ensure there is no potential conflict of interest that could draw into question the findings of the board. The charge of the DSMB will be to oversee the interim data from the study at selected intervals-intervals of time and enrollment-to ensure trial protocols are being followed, to monitor for adverse events, and to assess the completeness and timely reporting and accounting of data. Given the nature of our study, with its inability to blind clinicians and patients to the treatment arm patients are assigned, the DSMB will also serve as a monitor for possible confounding variables not initially adjusted for. The patient population for REVERSE involves, without exception, patients who are critically ill and the DSMB will importantly be charged with the assessment and implementation of the stopping criteria detailed in the Randomization section of the article. All meetings of the DSMB will be confidential, and protocols will be followed for the secure transmission of information and findings.

The CAC will also be constituted of experts in the fields of cardiogenic shock, mechanical circulatory support, biostatistics, and clinical trials. The members of the CAC will also be free of conflicts of interest. The data and trial information provided to the CAC will be blinded for their evaluation. Although the primary end points do not lend themselves to ambiguity, many of the secondary end points would benefit from a panel with expertise in the field to fairly delineate trial participants' performances for comparison. Their participation will determine safety and efficacy end points as having been met and will help define and ensure consistency and standardization in performance measures across the study. The CAC will adjudicate any untoward event as expected or unexpected, and define its severity. These will be communicated regularly to the institutional review board. As with the DSMB, the CAC will be charged with complete confidentiality in and of their discussions to protect our patients and the integrity of the REVERSE study. 